\title{
HIGH RESOLUTION NEAR-INFRARED IMAGING AND COMPUTER SIMULA- TIONS OF A LUNAR OCCULTATION OF THE GALACTIC CENTER
}

\author{
D. A. Simons, E. E. Becklin, and K. -W. Hodapp \\ University of Hawaii \\ Institute for Astronomy \\ 2680 Woodlawn Drive \\ Honolulu, Hawaii 96822
}

\begin{abstract}
A high resolution K-band $(2.2 \mu \mathrm{m})$ image of the Galactic center was acquired as part of an effort to develop computer simulations of a lunar occultation of the IRS 16 region that was previously observed in 1986 September. Through our computer simulations and recent observations of another occultation that occurred in 1988 September, confident identifications of the objects detected during the 1986 September occultation were acquired. In particular, IRS 16C, 16SW, and 16NE were all identified and found to be unresolved $\left(<0.05\right.$ " in angular extent). ${ }^{\dagger}$
\end{abstract}

\section{K-band Imaging}

Figure 1 is a high resolution K-band image of the Galactic center that was acquired at the $f / 35$ focus of the University of Hawaii's $2.2 \mathrm{~m}$ telescope on 1987 August 6, using the JPL/SISEX $64 \times 64$ infrared array (Capps et al. 1987). An exposure of $200 \mathrm{~s}$ was used to generate the image, which has a scale of $\sim 0.14$ arcsec pixel $^{-1}$, a limiting magnitude of $\sim 15$ mag $\operatorname{arcsec}^{-2}$, and a resolution of $\sim 0.7 "$. Figure 2 is a contour plot of the same image depicted in Figure 1 , with the detected infrared sources labeled. The 4 primary components of IRS 16 are clearly evident in Figures 1 and 2, as well as IRS 1, a portion of IRS 7, and diffuse emission.

\section{Fresnel Diffraction Analysis of the 1986 September Occultation Data}

The basic conditions of the 1986 September lunar occultation are described in Adams et al. (1988). The generally unfavorable occultation conditions, along with the relatively slow (20 ms) sampling rate used by Adams et al. (1988), made it impossible to actually resolve diffraction fringes in the data they acquired, meaning that it was impossible to determine accurate source sizes from their data, except for objects larger than $\sim 0.1 "$. Fresnel diffraction model fits to the occultation data, for a range of source sizes, did not lead to a statistically unique set of source sizes for the three compact objects detected during the occultation (called U1, U2, and U3 in Adams et al. 1988). Furthermore, an estimated $\pm 1 \mathrm{~s}$ error in timing predictions, together with uncertainties in source positions, made it difficult to confidently identify the objects detected during the occultation.

† The identification of IRS $16 \mathrm{NE}$ as an unresolved source differs from the oral results presented by the authors in July at UCLA. 


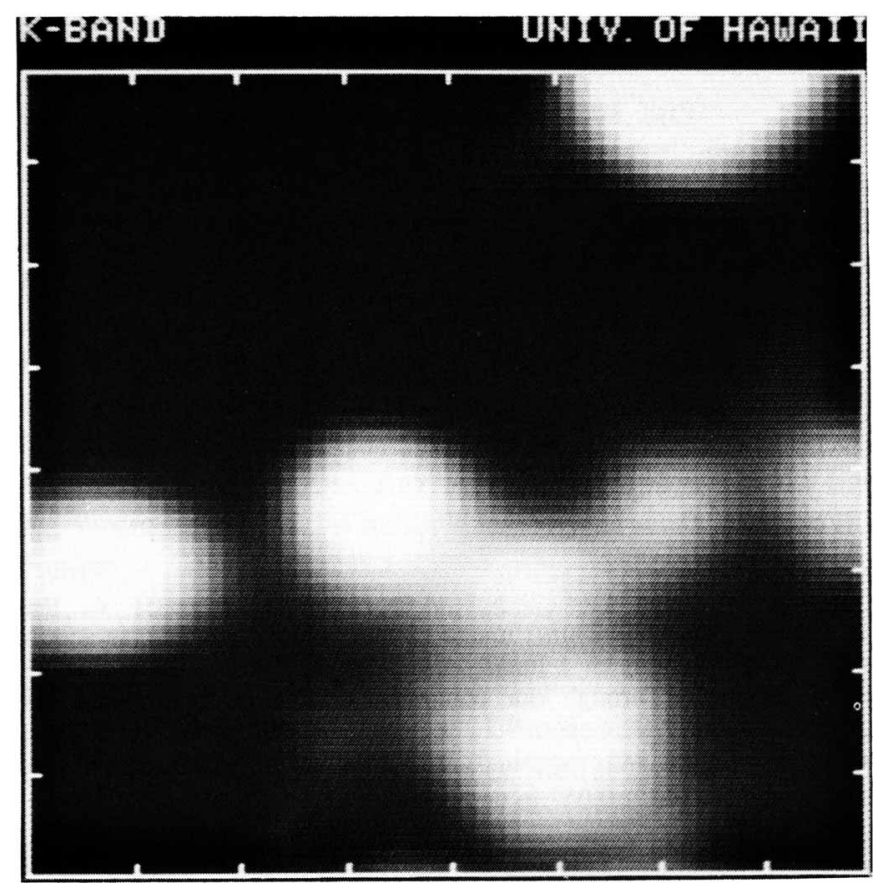

Figure 1. A $200 \mathrm{~s}$ K-band image of the IRS 16 region. North is up and East is to the left with tick marks at $1 "$ intervals. The seeing during the time this image was acquired was $\sim 0.7 "$.

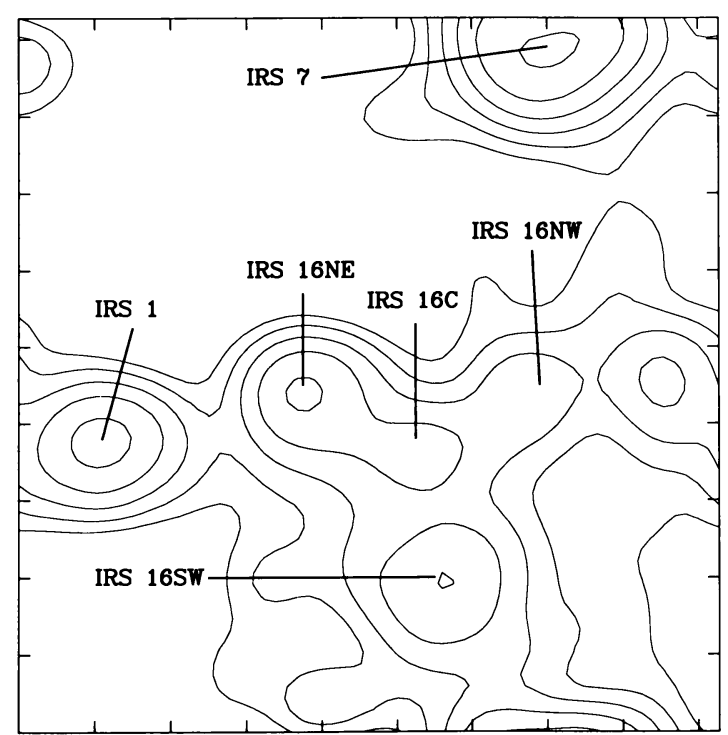

Figure 2. A contour plot of the same image depicted in Figure 1 is presented. Contours have been made at 0.5 mag steps with tick marks at 1 " intervals. The various components of IRS 16 are identified, as well as the nearby infrared sources IRS 1 and IRS 7. 


\section{Computer Simulations of the 1986 September Occultation}

Extensive computer simulations of the 1986 September lunar occultation data, based upon the previously described K-band image, were conducted to reduce the uncertainties left in the Adams et al. (1988) analysis. The computer algorithm used to perform the simulations was based upon a mathematical line (in the $y=m x+b$ sense) being swept west-to-east across the image. The slope of the line determined the limb angle of the simulation. The "motion" of the line (i.e., the $\Delta \mathbf{x}$ increments) was set to equal the amount that the moon moved during the 20 ms sampling periods used by Adams et al. (1988). While a particular line was formed by the program, the ADU values of the pixels which fell along the line were summed (i.e., a line integral was evaluated).

Before comparisons between model results and the occultation data could be attempted, the latter had to be differentiated, i.e. the differences between consecutive pairs of data points had to be evaluated, so that it also had the mathematical form of a set of line integrals. Subsequent to differentiation, the occultation data was smoothed to the same resolution as the K-band image. Because the model lines were spatially incremented by amounts that were equal to the distance the moon traveled during each sampling period, it was possible to directly "overlay" model values with occultation values in order to determine which possible "overlay" yielded the best agreement between model and occultation data sets. It was found that the model based upon a $30^{\circ}$ limb angle yielded a significantly better fit to the occultation data $(r \sim 0.99)$ than those based upon $0^{\circ}, 15^{\circ}$, and $45^{\circ}$ limb angles - in agreement with the predicted nominal limb angle of $28^{\circ}$.

Since model data points could be traced back to specific sources within the analyzed image, it was possible to associate particular events within the raw occultation data with objects in the Galactic center image. From the correspondence between sources in the image and events in the occultation, it is strongly suggested that event U1 corresponds to IRS $16 \mathrm{C}$, U2 corresponds to IRS 16SW, and U3 corresponds to IRS 16NE (see Figure 3). It should be noted that we recently observed another lunar occultation of IRS 16 using high speed infrared array imaging which yielded unambiguous source identifications that confirm our analysis of the Adams et al. (1988) data. We are tentatively associating the extended emission, identified as IRS 16NE in Adams et al. (1988), with diffuse flux 1" East of IRS 16SW. A much more complete description of our recently acquired data, as well as our analysis of the data acquired by Adams et al. (1988), will be given in a forthcoming publication.

\section{Conclusions}

Through high resolution near-infrared imaging of the Galactic center, extensive computer modeling, and recent observations of a lunar occultation of IRS 16 which occurred in 1988 September, we have identified the sources detected by Adams et al. (1988) in 1986 September. In particular, we have determined that IRS $16 \mathrm{C}, 16 \mathrm{SW}$, and $16 \mathrm{NE}$ were all detected by Adams et al. (1988) and have apparent angular sizes that are less than $\sim 0.05 "$. 


\section{Acknowledgements}

We thank the support staff, day crew, and night operators of the UH $2.2 \mathrm{~m}$ telescope for their assistance with the infrared imaging. We also thank the IRTF for the use of their computing facilities during the Fresnel diffraction modeling and occultation simulations. The SISEX IR array is the result of work by the JPL Infrared Technology Group in support of the Spaceborne Imaging Spectrometer Project Office at JPL under funding by the NASA office of Space Science and Applications. The IR array manufacturer is the Rockwell International Science Center.

\section{References}

Adams, D. J., Becklin, E. E., Jameson, R. F., Longmore, A. J., Sandqvist, Aa., and Valentijn, E. 1988, Ap. J. (Letters), 327, L65.

Capps, R. W., Hodapp, K. -W., Hall, D. N. B., Becklin, E. E., Simons, D. A., Bailey, G. C., and Wright, V. G. 1987, in Infrared Astronomy with Arrays, (Honolulu: University of Hawaii, Institute for Astronomy), ed C.G. Wynn-Williams and E.E. Becklin, p222.

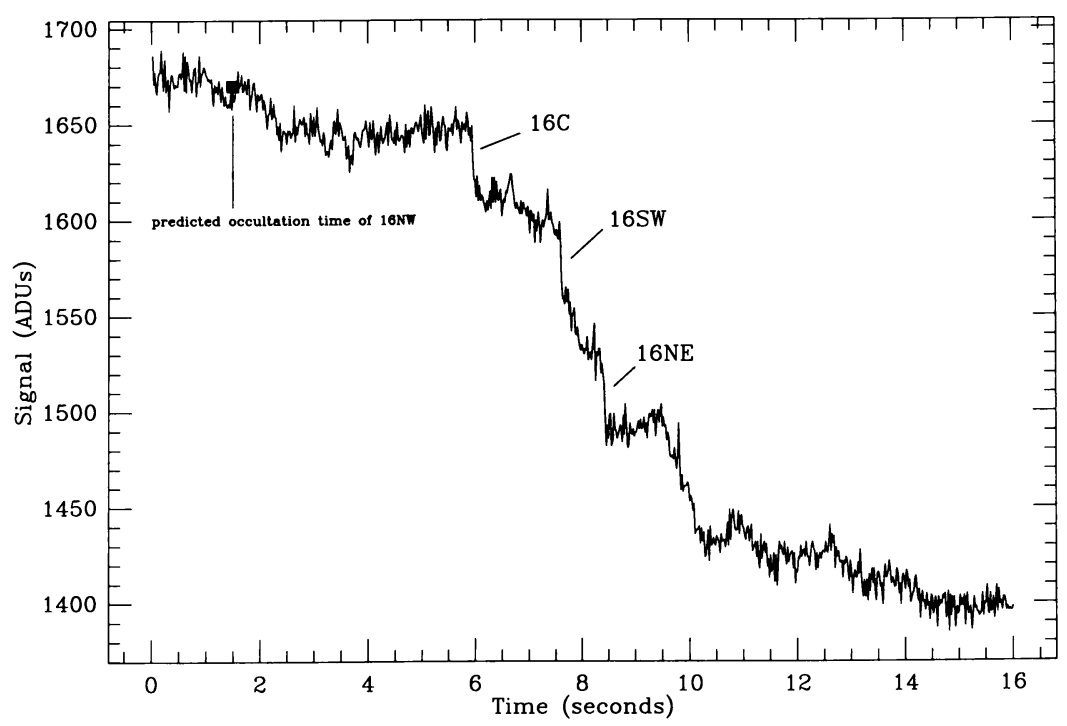

Figure 3. The raw occultation data acquired by Adams et al. (1988) is plotted, with the source identifications next to each event. The source identifications were derived from extensive computer simulations of the lunar occultation of the Galactic center, as it was observed by Adams et al. (1988) in 1986 September, as well as recent observations of another occultation of IRS 16 that occurred in 1988 September. 